\title{
Evaluation of Laparoscopy Combined with Intraoperative Gastroscopic Local Gastrectomy in the Treatment of Gastric Neuroendocrine Tumors
}

\author{
Nannan Zhao*, Xinxin He \\ Department of Gastroenterology, Affiliated Hospital of Chifeng University, Chifeng 024000, Inner Mongolia, China \\ *Corresponding author: Nannan Zhao, 1934720586@qq.com
}

\begin{abstract}
Objective: To analyze the clinical effect of laparoscopy combined with intraoperative gastroscopic local gastrectomy in the treatment of gastric neuroendocrine tumors. Methods: A total of 100 patients with gastric neuroendocrine tumors that were treated in the Affiliated Hospital of Chifeng University from January 2016 to March 2021 were selected as the research subjects. They were divided into two groups by the digital table method. The control group underwent laparoscopic partial gastrectomy while the research group underwent laparoscopy combined with intraoperative gastroscopic partial gastrectomy. The curative effects of the two groups were compared. Results: There was no significant difference in the number of surgical lymph node dissections between the two groups $(p>0.05)$; the amount of bleeding in the research group was lower than that in the control group; the operation time, time taken to get out of bed, gastrointestinal function recovery time, time taken for first eating, and hospital stay were shorter than those in the control group $(p<0.05)$; the incidence of complications and the recurrence rate in the research group were lower than those in the control group ( $p<0.05)$. Conclusion: Laparoscopy combined with intraoperative gastroscopic local gastrectomy in the treatment of gastric neuroendocrine tumors has significant clinical effect and high safety.
\end{abstract}

Keywords: Laparoscopy; Gastroscopy; Local gastrectomy; Clinical efficacy; Gastric neuroendocrine tumor

Publication date: September 2021; Online publication: September 30, 2021

\section{Introduction}

Neuroendocrine tumors are mainly heterogeneous tumor diseases occurring in neuroendocrine organs, which can occur in multiple organs or tissues. Among them, gastric neuroendocrine tumors are more common. This type of tumor mainly involves argyrophilic cells at the bottom of the gastric mucosal tube. Once suffering from the disease, patients do not usually show specific symptoms. They have certain similarities with gastric tumors, and they need to be treated in time, otherwise when distant metastasis develops, there would be more harm to affected patient ${ }^{[1]}$. At present, there are many clinical treatments for this disease, such as endoscopic submucosal dissection and mucosal resection, which can achieve certain curative effects, but the surgical requirements and technology are relatively high. In recent years, it has been found that laparoscopy combined with intraoperative gastroscopic local gastrectomy has a unique advantage and it is able to achieve excellent results. In regard to that, this study explores the therapeutic effect of laparoscopy combined with intraoperative gastroscopic local gastrectomy.

\section{Material and methods}

\subsection{Basic data}

In this study, 100 patients with gastric neuroendocrine tumors were selected from the Affiliated Hospital 
of Chifeng University from January 2016 to March 2021. The selected patients were divided into two groups by the digital table method. The control group comprised of 50 cases; the proportion of men to women was 25:25; the age range was 29 to 64 years old, with an average age of $50.37 \pm 1.58$; their average body mass index was $21.85 \pm 2.69 \mathrm{~kg} / \mathrm{m} 2$; the average tumor size was $5.84 \pm 1.51 \mathrm{~cm}$. The research group also comprised of 50 cases; the proportion of men to women was 26:24; the age range was 30 to 66 years old, with a mean of $50.41 \pm 1.61$; their average body mass index was $21.91 \pm 2.71 \mathrm{~kg} / \mathrm{m} 2$; the average tumor size was $5.86 \pm 1.49 \mathrm{~cm}$. There was no significant difference in the above data based on the calculation by a statistical software $(p>0.05)$.

Inclusion criteria: (1) met the criteria of gastric neuroendocrine tumors by biopsy and pathological diagnosis confirmation ${ }^{[2]}$; (2) flat abdomen without rebound pain and tenderness; (3) all patients were informed and voluntarily signed the study consent form; (4) approved by the hospital ethics committee. Exclusion criteria: (1) other malignant tumor diseases; (2) surgical contraindications; (3) mental illnesses and cognitive impairments.

\subsection{Methods}

For the research group, when laparoscopy combined with intraoperative gastroscopic partial resection was carried out, the patients were maintained in a supine position and general anesthesia was given. An incision was made at the lower end of each patient's umbilicus about $0.5 \mathrm{~cm}$, with an incision length of about $1 \mathrm{~cm}$, and a deliberate induction of carbon dioxide pneumoperitoneum. During the surgery, the gastroscope was accurately placed from the esophagus to the stomach under a detailed laparoscopic and gastroscopic monitoring. The specific location of the tumor was then determined. A laparoscope was used by the surgeon to mark the tumor envelope tissue. After the mark line was lifted, $45 \mathrm{~mm}$ and $65 \mathrm{~mm}$ linear cutting closures were used to remove the gastric neuroendocrine tumor. After successful removal, interrupted suturing was performed. Finally, the complete resection of the tumor was confirmed via gastroscopy. Without any active bleeding in the gastric cavity, the mirror was then withdrawn.

The patients in the control group underwent laparoscopic partial gastrectomy. The patients were first instructed to maintain a supine position and general anesthesia was given. The position of the incision and the pneumoperitoneum induction were consistent with that of the research group. The carbon dioxide pneumoperitoneum was reasonably controlled at $13 \mathrm{mmHg}$. Each patient's abdominal cavity was explored in detail by laparoscopy. After the position of the tumor had been determined, 1-2 stitches of suture under laparoscopy were made. The cutter was then closed with the help of an endoscopic linear cutting obturator. When the tumor had been completely contained, the cutter was closed without any bleeding noted.

The patients in the two groups required fasting, gastric acid inhibition, and rehydration. Only after preliminarily recovery, they were allowed to have liquid diet and anti-infection treatment in time.

\subsection{Observation indicators}

The surgeries of both the groups were observed, including the number of surgical lymph node dissections, operation time, and surgical bleeding. After operation, the time taken to get out of bed, gastrointestinal function recovery time, time taken for first eating, and the length of hospital stay were observed.

Postoperative complications such as surgical site infection, gastric motility disorders, and incision exudation were observed. The patients were followed up one year after discharge to determine the tumor recurrence.

\subsection{Statistical analysis}

Statistical Package for the Social Sciences (SPSS) version 23.0 was used for processing and analysis; t-test 
and chi-square test $\left(\mathrm{X}^{2}\right)$ were used for testing, $(\bar{x} \pm \mathrm{s})(\mathrm{n} / \%)$. There is a difference in the data if $p<0.05$.

\section{Results}

\subsection{Surgical treatment}

Based on the data in Table 1, there was no significant difference in the number of lymph node dissections between the two groups, $p>0.05$; the operation time of the patients in the research group was shorter than that of the control group; the amount of bleeding of the patients in the research group was lower than that of the control group $(p<0.05)$.

Table 1. Comparison of surgical treatment indexes $(\bar{x} \pm s)$

\begin{tabular}{ccccc}
\hline Group & $\mathrm{N}$ (example) & $\begin{array}{c}\text { Number of lymph node } \\
\text { dissections (piece) }\end{array}$ & Operation time (min) & $\begin{array}{c}\text { Surgical bleeding in } \\
\text { volume (ml) }\end{array}$ \\
\hline Control group & 50 & $34.15 \pm 13.57$ & $79.71 \pm 11.54$ & $64.12 \pm 18.45$ \\
Research group & 50 & $35.01 \pm 14.05$ & $58.46 \pm 10.71$ & $37.15 \pm 15.11$ \\
$\mathrm{t}$ & & 0.3113 & 9.5439 & 7.9968 \\
$p$ & & 0.7562 & 0.0000 & 0.0000 \\
\hline
\end{tabular}

\subsection{Postoperative recovery indicators}

The time taken to get out of bed, gastrointestinal function recovery time, time taken for first eating, and the length of hospital stay of the patients in the research group were shorter than those in the control group ( $p$ $<0.05$ ) (Table 2).

Table 2. Comparison of postoperative recovery indexes $(\bar{x} \pm \mathrm{s}, \mathrm{d})$

\begin{tabular}{cccccc}
\hline Group & N (example) & $\begin{array}{c}\text { Time taken to } \\
\text { get out of bed }\end{array}$ & $\begin{array}{c}\text { Gastrointestinal } \\
\text { function recovery time }\end{array}$ & $\begin{array}{c}\text { Time taken for } \\
\text { first eating }\end{array}$ & $\begin{array}{c}\text { Length of } \\
\text { hospital stay }\end{array}$ \\
\hline Control group & 50 & $3.12 \pm 0.98$ & $4.25 \pm 1.07$ & $5.42 \pm 0.98$ & $14.15 \pm 3.58$ \\
Research group & 50 & $2.64 \pm 0.75$ & $3.01 \pm 0.85$ & $4.25 \pm 0.87$ & $12.07 \pm 2.85$ \\
$\mathrm{t}$ & & 2.7504 & 6.4163 & 6.3132 & 3.2142 \\
$p$ & & 0.0071 & 0.0000 & 0.0000 & 0.0018 \\
\hline
\end{tabular}

\subsection{Postoperative complications}

The incidence of complications among the patients in the research group was lower than that in the control group $(p<0.05)$ (Table 3).

Table 3. Comparative complication rate (n /\%)

\begin{tabular}{cccccc}
\hline Group & N (example) & $\begin{array}{c}\text { Surgical site } \\
\text { infection }\end{array}$ & $\begin{array}{c}\text { Gastric motility } \\
\text { disorders }\end{array}$ & $\begin{array}{c}\text { Incision } \\
\text { exudation }\end{array}$ & Total incidence \\
\hline Control group & 50 & 4 & 1 & 2 & $7(14.00)$ \\
Research group & 50 & 1 & 0 & 0 & $1(2.00)$ \\
$\mathrm{X}^{2}$ & & & & & 4.8913 \\
$p$ & & & & & 0.0269 \\
\hline
\end{tabular}




\subsection{Follow-up recurrence rate}

One year after discharge, the follow-up survey found that four patients from the control group had recurrence, and the recurrence rate was $8.00 \%$ whereas all the patients in the research group recovered without any recurrence, and the recurrence rate was $0.00 \%$. The recurrence rate of the research group was lower than that of the control group $(p<0.05)$ with a significant difference $\left(X^{2}=4.1667, p=0.0412\right)$.

\section{Discussion}

Gastric neuroendocrine tumor is a tumor disease that is derived from neuroendocrine cells. Its degree of tumor differentiation is low, but it is a highly malignant tumor in nature. Lymph node metastasis or distant metastases may occur in the early stage, causing great harm to the patient's health; thus, it must be treated as soon as possible ${ }^{[3,4]}$. At this stage, surgical treatment is the first choice in clinical practice, mainly because the growth of gastric neuroendocrine tumors is relatively slow and most of these tumors have local infiltration and growth characteristics; thus, surgical resection is still the main treatment.

In the past, various investigations for these tumors were required before treatment. If the tumor does not involve the proper muscle layer and there is no ulceration, endoscopic mucosal dissection can be performed. In view of traditional open surgery, due to its large incision and surgical trauma, it increases the risk of infection and has high limitations. With the rapid development of minimally invasive concept and technology, laparoscopy has been gradually applied in clinical practice. Laparoscopy combined with gastroscopy for local gastrectomy has unique advantages. During the procedure, detailed observation and determination of the location of lesions can be made via gastroscopy. This can effectively prevent damage to other tissues due to blindness. At the same time, gastroscopy can be combined with surgery; it ensures complete resection of a focus and avoid missed minimally invasive surgical resections. In addition, it can also be used for specific types of tumors with high surgical safety ${ }^{[5-8]}$. Based on the results of this study, all surgical indexes of the research group were better than those of the control group. In addition, the postoperative complications and 1-year follow-up recurrence rate were lower among the patients in the research group compared to the control group. The difference in the data was significant.

In conclusion, laparoscopy combined with intraoperative gastroscopic local gastrectomy in the treatment of gastric neuroendocrine tumors has achieved significant clinical efficacy and high surgical safety.

\section{Disclosure statement}

The authors declare that there is no conflict of interest.

\section{References}

[1] Tan W, 2017, Effect of Laparoscopy Combined with Intraoperative Endoscopic Local Gastrectomy in the Treatment of Gastric Neuroendocrine Tumors. Chinese Journal of Practical Neurological Diseases, 4(110): 102-104.

[2] Er L, Wu M, Zheng X, et al., 2018, Value of Endoscopic Ultrasonography in Minimally Invasive Treatment of Gastrointestinal Neuroendocrine Tumors with Low Proliferative Activity. Clinical Meta-Analysis, (6): 515-518.

[3] Satoshi K, Manabu O, Satoshi I, et al., 2018, Laparoscopic Subtotal Gastrectomy with a New Marking Technique, Endoscopic Cautery Marking: Preservation of the Stomach in Patients with Upper Early Gastric Cancer. Surgical Endoscopy, : 1-7.

[4] Xiong Y, Liu X, Cheng C, et al., 2020, Clinical Features, Pathology and Prognosis of Primary Gastric Neuroendocrine Tumors. Chinese Journal of Internal Medicine, 59(04): 297-302. 
[5] Tsutomu, Namikawa, Sachi, et al., 2018, Endoscopic Ultrasonography Fine Needle Aspiration Biopsy Glomus Tumor Laparoscopic Gastrectomy. Oncology Letters, 17(1): 514-517.

[6] Zou B, Ning N, Xu J, et al., 2015, Clinical Comparison of Double Mirror Combined with Simple Laparoscopic Surgery in the Treatment of Benign and Malignant Gastric Tumors. Progress of Modern General Surgery in China, 18(006): 484-485, 493.

[7] Xie J, Huang C, Zheng C, et al., 2016, Evaluation of Laparoscopic Radical Gastrectomy in the Treatment of Gastric Neuroendocrine Carcinoma. Chinese Journal of Gastrointestinal Surgery, (19): 911.

[8] Qi L, Liu L, Zhu J, et al., 2018, Comparison of Endoscopic Submucosal Dissection and Mucosal Resection in the Treatment of Gastric Neuroendocrine Tumors. Journal of China Japan Friendship Hospital, 32(02): 28-30.

[9] Wang C, Zhang J, Zhang Z, et al., 2019, Clinicopathological Features and Prognosis of 117 Cases of Gastric Neuroendocrine Tumors. Chinese Journal of Oncology, 46(5): 33-40. 